Bangladesh J. Bot. 48(4): 989-1000, 2019 (December)

\title{
DETECTION AND ANALYSIS OF FREE AMINO ACIDS IN WAXY MAIZE DH LINES BY HPLC IN NORTH-EAST CHINA
}

\author{
Long Jiang, Haiyan Yu, Dianyuan Chen, Xiaoming Yu, Hui Xu, \\ Xue Ma, Bo Sun and Xin Mu Fan \\ College of Agronomy, Jilin Agricultural Science and Technology University, \\ Jilin, Jilin 132101, China
}

Keywords: Free amino acids, HPLC, Germplasm evaluation, Waxy maize DH lines

\begin{abstract}
Composition of free amino acid in waxy maize DH lines was analysed and the nutritional quality of waxy maize germplasm resources was evaluated by o-phthaladehyde (OPA) pre-column derivatization reversed-phase high performance liquid chromatography (HPLC). Results obtained showed that there were at least 14 kinds of free amino acids, DH57 line had the highest $(25.185 \mathrm{mg} / \mathrm{g})$, and DH21 line had the lowest $(6.203 \mathrm{mg} / \mathrm{g})$ and the average was $12.226 \mathrm{mg} / \mathrm{g}$. The contents of essential amino acids varied from 2.59 to $14.09 \mathrm{mg} / \mathrm{g}$, accounting for $29.55-75.64 \%$ of the total free amino acids. The quality of free amino acids was evaluated by comprehensive indices with principal component analysis and the first top three DH lines were DH57, DH59 and DH55. The contents of free amino acids in 60 waxy maize DH lines had obvious genetic diversity. As to the free amino acid content, the amino acids quality of DH57, DH59 and DH55 were the best, while DH21 was the worst. Results provide a theoretical guidance for the cultivation of new varieties of waxy maize with good quality.
\end{abstract}

\section{Introduction}

At present, seed companies in China regard haploid technology as the main breeding technology of maize inbred lines, which have established a special "DH production department" for the large-scale production of homozygous inbred lines in the commercial maize breeding system (Jiang et al. 2014, Dong et al. 2015). Fresh waxy maize not only tastes well but also has rich nutrition. With development of economy and improvement of living standards, the demand for fresh waxy maize grows rapidly in China, which has developed into a new industry (Shi et al. 2002). China is the origin of waxy maize and has rich germplasm resources (Huang and Rong 1998). In China, it had carried out the maize DH breeding in order to speed up the cultivation of excellent waxy inbred lines and applied to waxy maize breeding practice. Many studies had shown that it was likely possible that fresh waxy maize with good nutrition and health care became the staple cereal, resulting in a boom of waxy maize DH breeding (Gong et al. 2012). Waxy maize germplasm was the basic material to select new varieties and occupied a pivotal position in the waxy maize DH breeding process.

Free amino acids (FAA) contained various essential amino acids, which cannot be synthesized by human, and also flavour amino acids, which were important indicators to evaluate food nutritional quality. There were a lot of studies on free amino acids. Yang and Sun determined composition of free amino acids in litchi and apple and evaluated the fruit quality of different varieties (Yang et al. 2011, Sun et al. 2012). Li et al. (2011) analysed the composition and content of free amino acids in different types of tobacco and Porphyra yezoensis. It has become a public awareness that enhancement of essential amino acid contents and health benefit components in grain crops by genetic engineering and protein design can improve nutritional quality (Beauregard

*Author for correspondence:<jlnykjxyjl@163.com>. 
and Hefford 2006, Wenefrida et al. 2009, Galili and Amir 2013). Enrichment of some essential amino acids, such as lysine (Lys), tryptophan (Trp), and methionine (Met) has been successful in maize (Galili and Amir 2013).

The free amino acids content in maize grain was determined by Wang et al. (2010) using HPLC method. Hao et al. (2008) measured the content of amino acid in fresh sweet and glutinous maize grains, and their results provided a reference for waxy maize breeding. There were some reports on the changes of free amino acids content in maize grains of hybrids (Caňas et al. 2009, Seebauer et al. 2004). Caňas et al. found that under low $\mathrm{N}$ fertilization conditions, maize Io line accumulated glutamine, asparagine and alanine preferentially in the developing kernels, whereas in maize $\mathrm{F}_{2}$ line, glutamine and proline were the predominant amino acids (Caňas et al. 2009). Seebauer et al. found that the major amino acids in the cob of maize were glutamine (Gln), aspartic acid (Asp), asparagine (Asn), glutamate, and alanine. Gln concentrations dropped dramatically from 2 to $14 \mathrm{~d}$ after silking in both pollinated and unpollinated cobs, whereas all other measured amino acids accumulated over time in unpollinated spikelets and cobs, especially Asn (Seebauer et al. 2004). However, little research has been conducted on free amino acids in waxy maize inbred lines.

Currently, several new fresh waxy maize varieties are being bred in China. Although most of the waxy maize varieties have met certification requirements, a few had excellent quality. National and regional reports indicated that how to improve eating quality of waxy maize is the main task of the current waxy maize breeding (Zhang et al. 2008). Therefore, it is urgent for breeders to continue to find extra waxy maize germplasm resources, to evaluate and utilize existing inbred lines properly and to cultivate new waxy maize varieties. The present researchers used o-phthaladehyde (OPA) pre-column derivatization reversed phase high performance liquid chromatography (RP-HPLC) to detect the composition of free amino acids in 60 waxy maize inbred lines and screen waxy maize DH lines with excellent quality through analysis and evaluate their nutritional and eating quality. Results obtained would provide a theoretical guidance for the cultivation of new varieties of waxy maize with good quality.

\section{Materials and Methods}

Sixty waxy maize DH lines provided by Jilin Agricultural Science and Technology University special maize breeding research group were used for this experiment, which were planted in Jilin Maize Breeding Base of Jilin Agricultural Science and Technology University in May 2017. In August, 20 grains from middle ear of good grout, dried naturally and pest-free maize inbred lines were selected and put into oven at $40^{\circ} \mathrm{C}$ for $24 \mathrm{hrs}$. The grains were crushed into powder by grinder and passed through a 60-mesh standard sieve and kept in sealed bags (Liu et al. 2007). Reagents used were ethanol, acetic acid, sodium hydroxide, sodium dihydrogen phosphate, sodium tetraborate, phthalaldehyde and mercaptoethanol of analytical grade. Acetonitrile and methanol (Sigma, USA) were of HPLC grade. Ultrapure water was prepared by Milli-Q ultrapure water system (Millipore, USA).

Dionex U3000 high performance liquid chromatography system including DGP-3600 pump, WPS-3000 auto sampler, TCC-3100 automatic temperature control oven, DAD-3000 detector and a chameleon chromatography workstation were used. AUW220 electronic balance (Daojin, Japan), HWS-26 electric heated water bath (Shanghai, China), KQ-100DE CNC ultrasonic cleaner (Kunshan Ultrasonic Instrument Co., Ltd, Kunshan, China) and Sigma3-30k high-speed refrigerated centrifuge (Sigma, USA) were used.

The column was Agilent Eclipse XDB-C18 column $(5 \mu \mathrm{m}, 250 \mathrm{~mm} \times 4.6 \mathrm{~mm})$, the column temperature was $40^{\circ} \mathrm{C}$, the detection wavelength was $338 \mathrm{~nm}$ and the flow rate was $1 \mathrm{ml} / \mathrm{min}$. 
Mobile phase Buffer A was methanol: acetonitrile: water $=46: 46: 10(\mathrm{v} / \mathrm{v} / \mathrm{v})$; mobile phase buffer $\mathrm{B}$ was $10 \mathrm{mM}$ sodium dehydrogenate phosphate $(\mathrm{pH} 7.5)$. The following elution program was applied:100\% buffer B, 0 min; 82\% buffer B, 10 min; 76\% buffer B, 15 min; $59 \%$ buffer B, 21 min; $57.8 \%$ buffer B, $23 \mathrm{~min}$; $42 \%$ buffer B, $25 \mathrm{~min} ; 41 \%$ buffer B, $27 \mathrm{~min}$ and $100 \%$ buffer B, 31 $\min$.

Five microliter borate buffers $(\mathrm{pH} 9.5)$ were drawn by an automatic syringe, then $1.0 \mu \mathrm{l}$ o-phthaladehyde (OPA) was drawn and the syringe was washed once. Two microliter sample was drawn, and the syringe was washed once. The sample was mixed eight times in situ and after 90 sec, the simple was injected.

The present workers accurately weighed $0.3 \mathrm{~g}$ sample and mixed it with same volume of distilled water and loaded it into test tube. The sample was subjected to sonication treatment for 30 $\mathrm{min}$ at room temperature, and centrifuged at $12000 \mathrm{r} / \mathrm{min}$ at $4^{\circ} \mathrm{C}$ for $15 \mathrm{~min}$. The residue was extracted once again. The two supernatants were combined and filtered through a $0.22 \mu \mathrm{m}$ Millipore nylon. The sample was stored in $-20^{\circ} \mathrm{C}$ and analysed by o-phthaladehyde (OPA) pre-column derivatization reversed phase HPLC.

According to retention time of 16 kinds of amino acid standards, the types of amino acids were determined. Using a series of concentrations of amino acid standard as abscissa and peak area as vertical axis, linear equations of 16 kinds of amino acids were obtained and free amino acid contents of samples were calculated using area normalization method (Peter et al. 1979).

Principal amino acids components were analysed by SPSS20 software package (www.spss.com/statistics) according to the following formula:

$\mathrm{I}=\mathrm{a}_{1} \mathrm{y}_{1}+\mathrm{a}_{2} \mathrm{y}_{2} \ldots+\mathrm{a}_{\mathrm{m}} \mathrm{y}_{\mathrm{m}}$,

where I represents comprehensive evaluation index of sample; $a_{m}$ presents variance contribution of main component; $y_{m}$ presents every main component value of each sample.

\section{Results and Discussion}

Under the chromatographic conditions described above, a series of different concentrations of mixed solution of 16 kinds of amino acids standards were measured and their standard chromatograms were obtained (Fig. 1). To obtain the linear equations of 16 kinds of amino acids, the peak area $\mathrm{Y}$ against the corresponding concentration $\mathrm{X}(\mu \mathrm{g} / \mathrm{ml})$ to establish standard curves (Table 1). The results showed that the detected concentrations were in a good linear relationship and all the correlation coefficients (r) were greater than 0.9990 .

Free amino acids in a sample through OPA in situ derivatization were separated and detected by RP-HPLC, and their chromatogram peaks were shown in Fig. 2. Qualitative analysis showed that 16 kinds of free amino acids were detected in the samples including seven kinds of essential amino acids and 9 kinds of non-essential amino acids. The results of free amino acids content showed that at least 14 kinds of amino acids were detected in 60 waxy maize DH lines (Table 2). DH57 line had the highest free amino acids content $(25.185 \mathrm{mg} / \mathrm{g})$, while DH21 line had the lowest free amino acids content $(6.203 \mathrm{mg} / \mathrm{g})$. The average free amino acids content for 60 waxy corn inbred lines was $12.226 \mathrm{mg} / \mathrm{g}$. The free amino acids contents in $24 \mathrm{DH}$ lines were higher than the average value. As to composition of free amino acids in the samples, Asp, Glu, Ser and Tyr were the top four amino acids. The total content of these four amino acids was between 3.205 and $19.603 \mathrm{mg} / \mathrm{g}$, accounting for $30.88-79.84 \%$ of the total content of the free amino acids. Thr content was the lowest, and was not detected in the majority of samples. 
Table 1. The regression analysis of $\mathbf{1 6}$ standard amino acids of waxy maize DH lines.

\begin{tabular}{lcc}
\hline Amino acids & Linear equation & Correlation \\
\hline Asp & $\mathrm{y}=0.0068 \mathrm{x}+0.0124$ & 0.9993 \\
Glu & $\mathrm{y}=0.0125 \mathrm{x}+0.0176$ & 0.9989 \\
Ser & $\mathrm{y}=0.0373 \mathrm{x}-0.0688$ & 0.9992 \\
His & $\mathrm{y}=0.0215 \mathrm{x}-0.0287$ & 0.9994 \\
Gly & $\mathrm{y}=0.0733 \mathrm{x}-0.1247$ & 0.9993 \\
Thr & $\mathrm{y}=0.0348 \mathrm{x}-0.0353$ & 0.9992 \\
Ala & $\mathrm{y}=0.0375 \mathrm{x}-0.1047$ & 0.9990 \\
Arg & $\mathrm{y}=0.0288 \mathrm{x}-0.0744$ & 0.9995 \\
Cys & $\mathrm{y}=0.0307 \mathrm{x}-0.0450$ & 0.9994 \\
Tyr & $\mathrm{y}=0.0083 \mathrm{x}+0.0667$ & 0.9989 \\
Val & $\mathrm{y}=0.0166 \mathrm{x}-0.0274$ & 0.9991 \\
Met & $\mathrm{y}=0.0687 \mathrm{x}+0.0330$ & 0.9994 \\
Phe & $\mathrm{y}=0.0241 \mathrm{x}-0.0389$ & 0.9993 \\
Iso & $\mathrm{y}=0.0273 \mathrm{x}-0.0159$ & 0.9995 \\
Leu & $\mathrm{y}=0.0568 \mathrm{x}-0.0159$ & 0.9997 \\
Lys & $\mathrm{y}=0.0336 \mathrm{x}-0.1640$ & 0.9992 \\
\hline
\end{tabular}

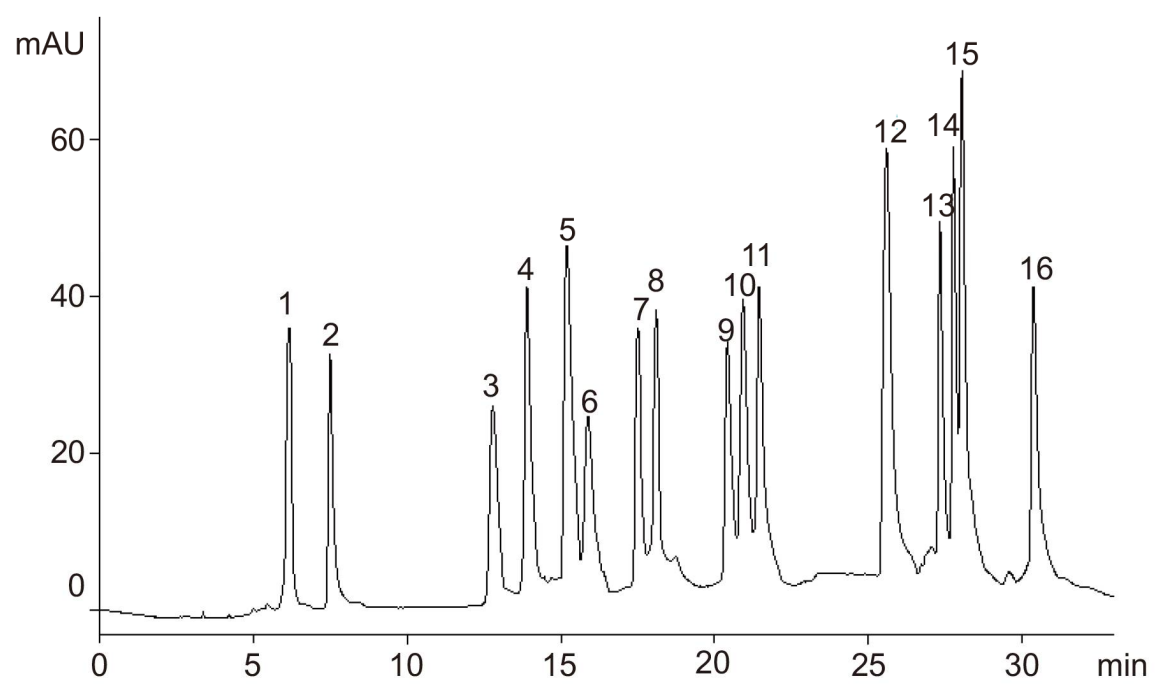

Fig. 1. A HPLC chromatogram of amino acid standards. 1. Asp 2. Glu 3. Ser 4. His 5. Gly 6. Thr 7. Ala 8. Arg 9. Cys 10. Tyr 11. Val 12. Met 13. Phe 14. Ile 15. Leu 16. Lys. 
Free amino acids content varied largely in different waxy maize DH lines from 0.000 to 8.727 $\mathrm{mg} / \mathrm{g}$ (Table 3). Thr and Ala had greater variability and the variation coefficient of them was greater than 100\%. Arg, Cys, Val, Asp, Tyr, Ser, Met and Lys were in the middle and the variation coefficient of them ranged from 50 to $100 \%$. Glu, Gly, His, Leu, Phe and Ile had lower variability and the variation coefficient of them was less than 50\%. The types of free amino acids in waxy maize inbred lines were significantly different.

With the continuous improvement of people's living standard, people are increasingly concerned about the nutritional quality and eating quality of waxy maize. Improvement of eating quality of waxy maize is the main task of the current waxy maize DH breeding (Galili and Amir 2013). Previously, the inheritance of free amino acids in maize was studied and the breeders could select some inbred lines with high content of free amino acids as parents to nurture new varieties with high amino acid content. Ke et al. (2006) found that the genetic traits of maize amino acids showed the following characteristics: Isoleucine, arginine and glycine contents were mainly influenced by additive gene effect; Lysine, methionine and aspartic content were influenced by both additive effect and non-additive effect; The remaining ten kinds of amino acids content were mainly affected by non-additive effect; Proline, phenylalanine, valine, leucine and alanine had relatively high broad heritability, while isoleucine, arginine and glycine had relatively high narrow heritability. Qi et al. (2001) also found that to improve lysine content in maize grain, parents must have high lysine content. To increase methionine content, difference in methionine content between parent inbred lines should not be too large and high content in both parent lines was the best method.

Those amino acids which cannot be synthesized by human (or other invertebrates) or their synthetic speeds are far from body need and must be provided by food proteins are called essential amino acids including Ile, Leu, Lys, Thr, Val, Phe and Met. The content of essential amino acids (EAA) in waxy maize DH lines was between $2.71 \mathrm{mg} / \mathrm{g}$ and $14.21 \mathrm{mg} / \mathrm{g}$, accounting for 29.67 $75.76 \%$ of the total free amino acids. The average value of them was $6.09 \mathrm{mg} / \mathrm{g}$ (Table 2). The content of each essential amino acid was also different in different waxy maize DH lines. DH59 line had the highest Ile and Met contents. DH55 line had the highest Leu content. DH58 line had the highest Lys, Thr, Val and Phe contents. Although human body can synthesize His and Arg, their synthetic speeds usually can't meet the normal need. Therefore, they were also called semi-essential amino acids or conditionally essential amino acids and were essential amino acids for the growth of children. In DH lines, the semi-essential amino acids content was between 0 and $2.21 \mathrm{mg} / \mathrm{g}$, accounting for 0 to $14.03 \%$ of total free amino acids. The average content of them was $0.98 \mathrm{mg} / \mathrm{g}$. DH36 line had the highest His content. DH16 line had the highest Arg content. The amino acids which can be synthesized from simple precursors by human (or other vertebrate) body do not need to be obtained from food are called non-essential amino acids. The content of non-essential amino acid was between 2.95 and $14.39 \mathrm{mg} / \mathrm{g}$ in waxy maize DH lines.

Foods contained some flavour amino acids, i.e. Glu, Asp, Gly and Ala. Those can interact with a variety of taste receptors in the mouth, so it can make food delicious. Umami amino acids included Glu and Asp. Sweet amino acids included Ala, Gly and Ser. Aromatic amino acids included Tyr and Phe. Waxy maize's taste was very important, in which flavor amino acids played an important role. Analysis showed that of the total free amino acids, umami amino acid content in waxy maize DH lines was between 0.887 and $9.619 \mathrm{mg} / \mathrm{g}$, accounting for $6.09-47.48 \%$ and the average content was $3.37 \mathrm{mg} / \mathrm{g}$ (Table 2), sweet amino acid content was between 0.736 and 4.240 $\mathrm{mg} / \mathrm{g}$, accounting for $4.97-30.84 \%$ and the average content was $1.97 \mathrm{mg} / \mathrm{g}$ (Table2), aromatic amino acid content was between 0.784 and $9.389 \mathrm{mg} / \mathrm{g}$, accounting for $8.61-48.28 \%$ and the average content was $3.23 \mathrm{mg} / \mathrm{g}$ (Table 2). 
क

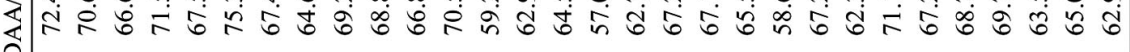

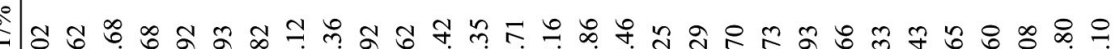

ஓं

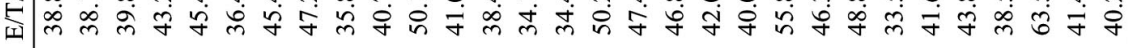

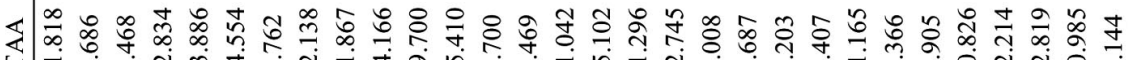

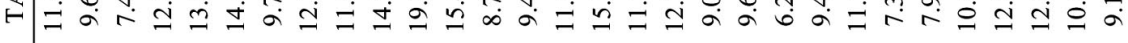

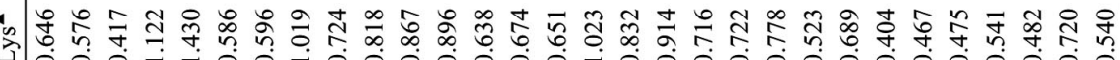

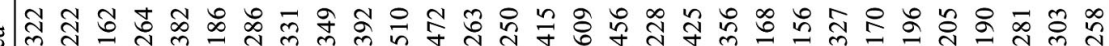

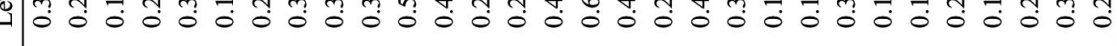

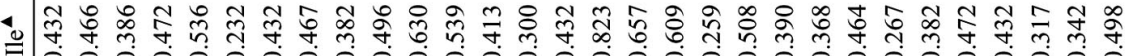

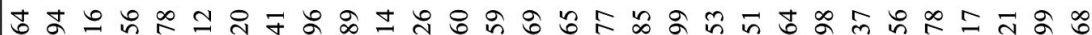

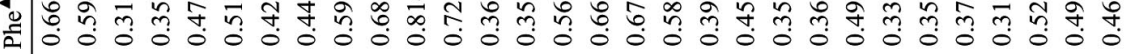

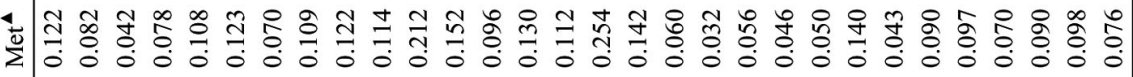

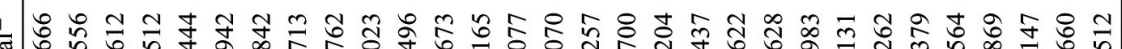

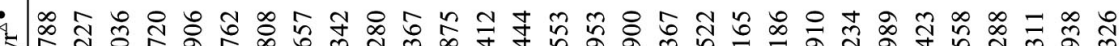

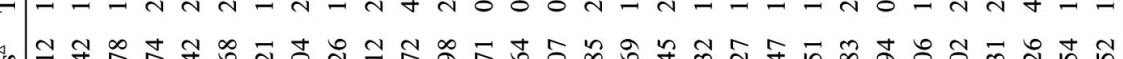

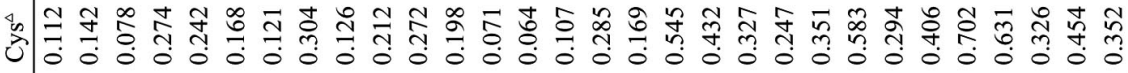
*

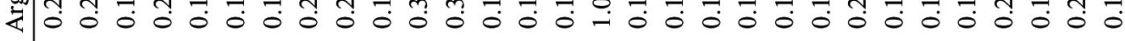

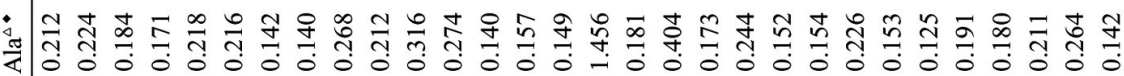

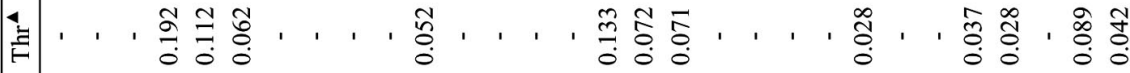

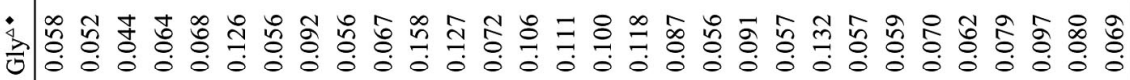

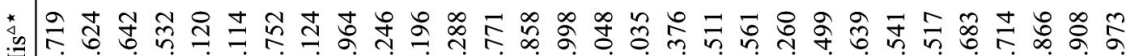

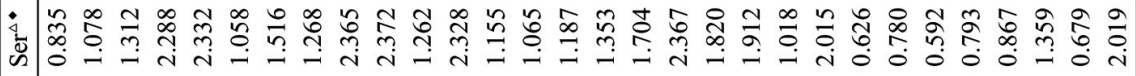

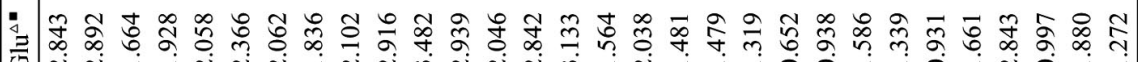

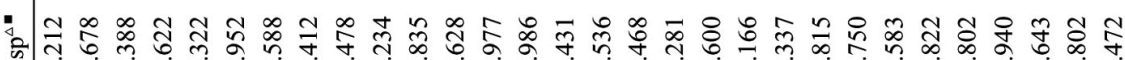
勾

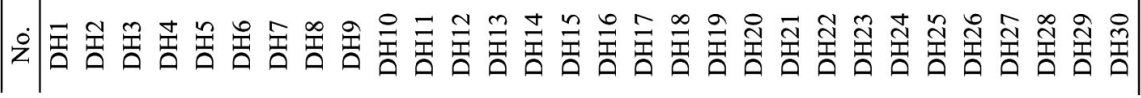




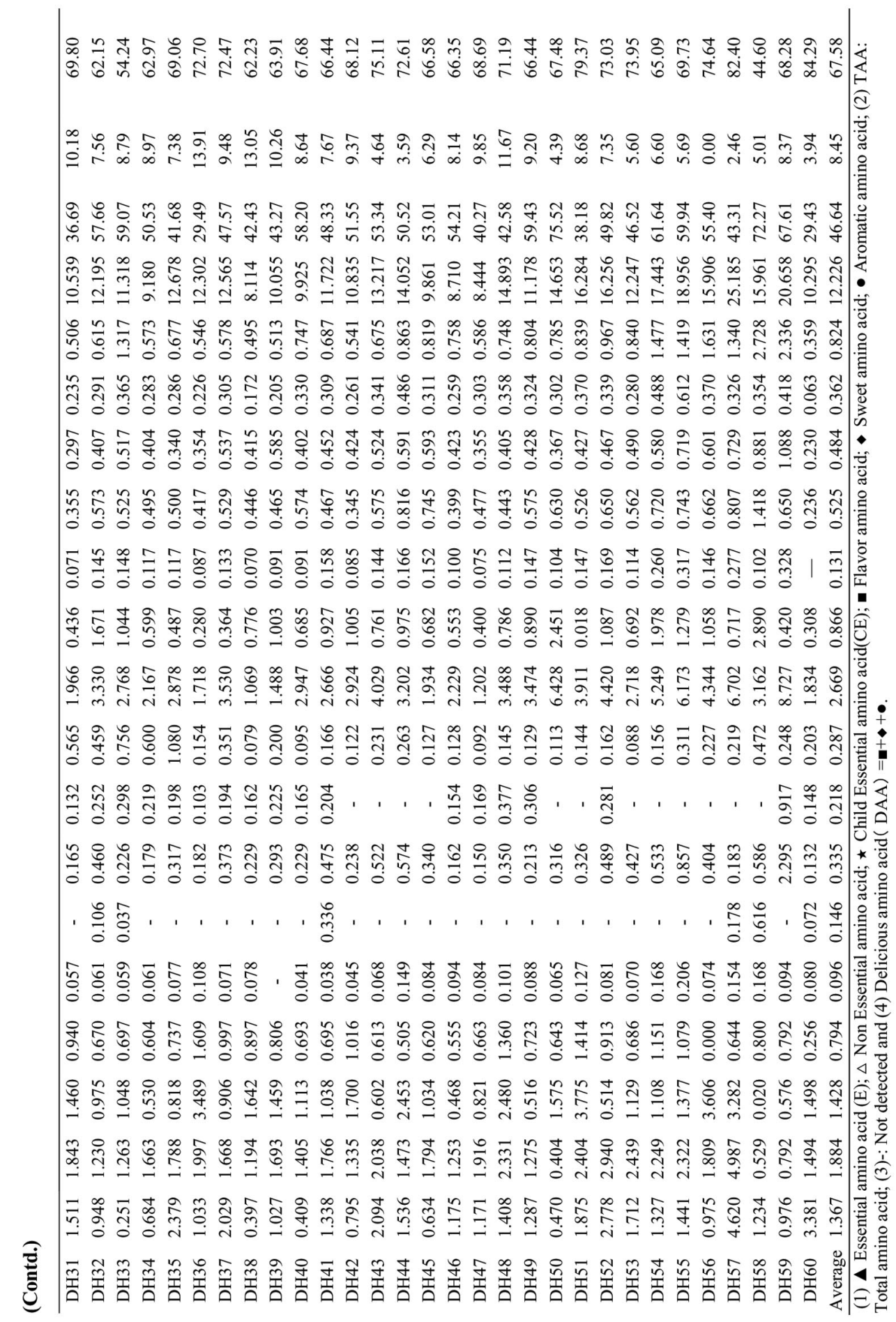


Sixteen kinds of free amino acids were comprehensively analysed in 60 waxy maize DH lines. Each amino acid content and total amount of all kinds of free amino acids were highest in DH57, DH59 and DH55 lines (Table 2). This result was consistent with the overall score result, indicating that the nutritional and eating quality of the three inbred lines were optimal. The result that DH21 had the lowest score was consistent with the fact that its free amino acids content was the lowest, indicating that amino acids quality in DH21 was poor. Currently, the ear taste identification for waxy maize DH breeding process was usually carried out after the hybrids were planted and seldom carried out in inbred lines. The artificial taste identification was not only affected by the food quality, but also by the people's taste preferences. Those results were largely influenced by human error (Liu et al. 2009), resulting in unclear direction of the genetic improvement of waxy maize. By measuring and analysis of flavour amino acids in DH lines, combining the eating quality with artificial tasting of DH lines can significantly improve the efficiency of waxy maize DH breeding.

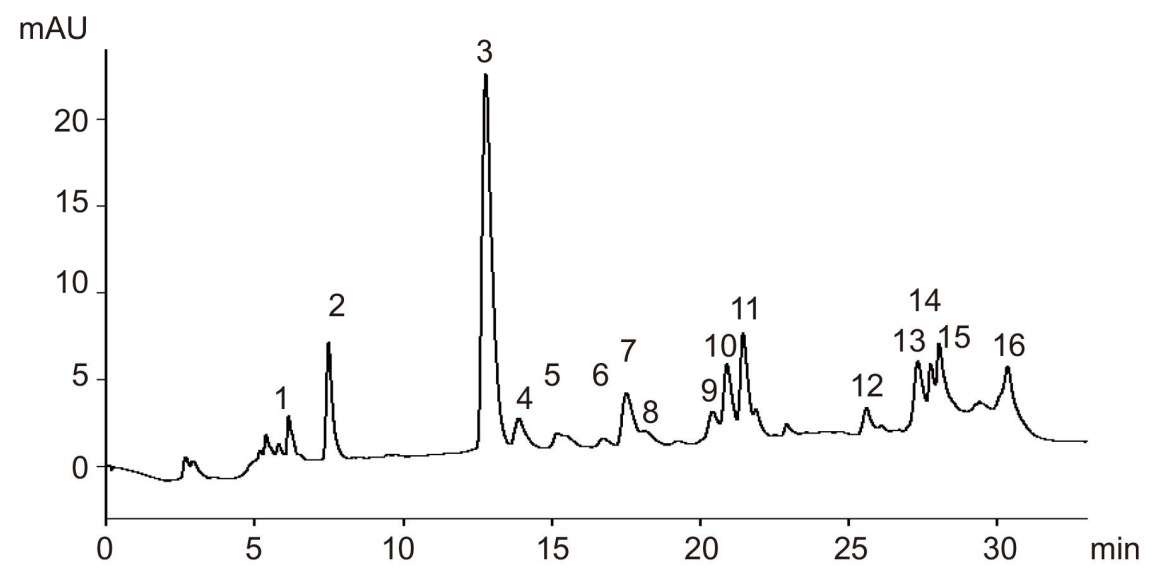

Fig. 2. A HPLC chromatogram of free amino acids in the sample. 1. Asp 2, Glu 3, Ser 4, His 5, Gly 6, Thr 7, Ala 8, Arg 9, Cys 10, Tyr 11, Val 12, Met 13, Phe 14, Ile 15, Leu 16. Lys.

Detected 16 kinds of free amino acids were chosen and SPSS20 software was used to analyse principal component of their main contents, and obtained 16 principal components which represent $100 \%$ of all the original information.

The first principal component was PC1 (51.68\%), the second principal component was PC2 (20.28\%) and the third principal component was PC3 (12.49\%), the above three principal components contained $84.22 \%$ of all the original information, which were usually considered to represent the majority of original information (Massart et al. 1997). Thus, the present investigators decided to extract the main ingredients from these three principal components. The first principal component included eight indicators: $\mathrm{PC} 1=0.472 \mathrm{Gly}+0.597 \mathrm{Ala}+0.989 \mathrm{Tyr}+0.769$ Met +0.498 Phe $+0.574 \mathrm{Ile}+0.466$ Leu +0.612 Lys; the second principal component included two indicators PC2 $=0.768 \mathrm{Asp}+0.848 \mathrm{Glu}$, the third principal component included one indicator PC3 $=0.882$ Ser. When using the principal component to make a comprehensive evaluation, main ingredients can be selected, namely $\mathrm{Y}_{1}, \mathrm{Y}_{2} \ldots \mathrm{Y}_{\mathrm{m}}$, with variance contribution rate of each principal component as weights to construct comprehensive evaluation index. In this paper, according to the three extracted principal components, investigators calculated the comprehensive evaluation indexes of different waxy maize DH lines (Table 4). Usually the larger the index, the better the 
comprehensive quality (Wang and Yang 2010). The order of the comprehensive evaluation indexes of three inbred lines was DH57 > DH59 > DH55, they were greater than 1. This result showed that the comprehensive evaluations index of three waxy maize $\mathrm{DH}$ lines were the best; the comprehensive evaluation index of DH21 was the lowest.

Table 3. Variance of amino acid content of different waxy maize inbred lines (mg/g).

\begin{tabular}{ccccccc}
\hline & Min. & Max. & Extreme & Average & Sd & CV/\% \\
\hline Asp & 0.248 & 4.624 & 4.368 & 1.378 & 0.854 & 62.028 \\
Glu & 0.403 & 4.878 & 4.584 & 1.876 & 0.768 & 41.946 \\
Ser & 0.022 & 3.757 & 3.756 & 1.448 & 0.816 & 56.746 \\
His & 0.000 & 1.612 & 1.612 & 0.796 & 0.296 & 37.060 \\
Gly & 0.042 & 0.208 & 0.164 & 0.084 & 0.045 & 40.698 \\
Thr & 0.000 & 0.616 & 0.626 & 0.048 & 0.098 & 255.262 \\
Ala & 0.132 & 2.294 & 2.172 & 0.335 & 0.336 & 102.482 \\
Arg & 0.000 & 1.046 & 1.065 & 0.183 & 0.178 & 97.822 \\
Cys & 0.062 & 1.080 & 1.026 & 0.287 & 0.212 & 72.543 \\
Tyr & 0.414 & 8.727 & 8.325 & 2.698 & 1.602 & 59.284 \\
Val & 0.028 & 2.892 & 2.874 & 0.886 & 0.556 & 62.538 \\
Met & 0.000 & 0.338 & 0.338 & 0.129 & 0.068 & 53.782 \\
Phe & 0.242 & 1.422 & 1.182 & 0.545 & 0.182 & 33.842 \\
Ile & 0.230 & 1.088 & 0.868 & 0.474 & 0.156 & 32.708 \\
Leu & 0.060 & 0.612 & 0.552 & 0.312 & 0.114 & 33.964 \\
Lys & 0.362 & 2.734 & 2.379 & 0.820 & 0.436 & 51.952 \\
Total & 6.405 & 25.175 & 18.762 & 12.246 & 3.522 & 28.714 \\
\hline
\end{tabular}

Through analyzing types and contents of free amino acid in grains of 60 waxy maize DH lines, the following conclusions can be made: (1) Sixteen kinds of free amino acids were detected in waxy maize DH lines including seven kinds of essential amino acids and nine kinds of non-essential amino acids. At least 14 kinds of amino acids were detected, and the variation coefficients of ten kinds of amino acid were greater than $50 \%$. The total content of free amino acids was between 6.203 and $25.185 \mathrm{mg} / \mathrm{g}$, showing a significant genetic diversity in each $\mathrm{DH}$ line. (2) Of the total free amino acids, umami amino acids content accounted for $6.09-47.48 \%$, sweet amino acids content accounted for $4.97-30.84 \%$ and aromatic amino acids content accounted for $8.71-48.28 \%$. Those results indicated that the nutritional quality and eating quality were different in each inbred line. (3) The method of principal component analysis was used to comprehensively evaluate $60 \mathrm{DH}$ lines and construct comprehensive evaluation index, $23 \mathrm{DH}$ lines were higher than the average score and the rest of $\mathrm{DH}$ lines were close to the average score, in which scores of DH57, DH59 and DH55 lines were outstanding. (4) Based on the analysis of the total content of free amino acids, essential amino acids content, flavor amino acids content and the main component of comprehensive evaluation score, it was concluded that DH57, DH55 and DH59 had the most optimal quality, while DH21 had the worst quality. 


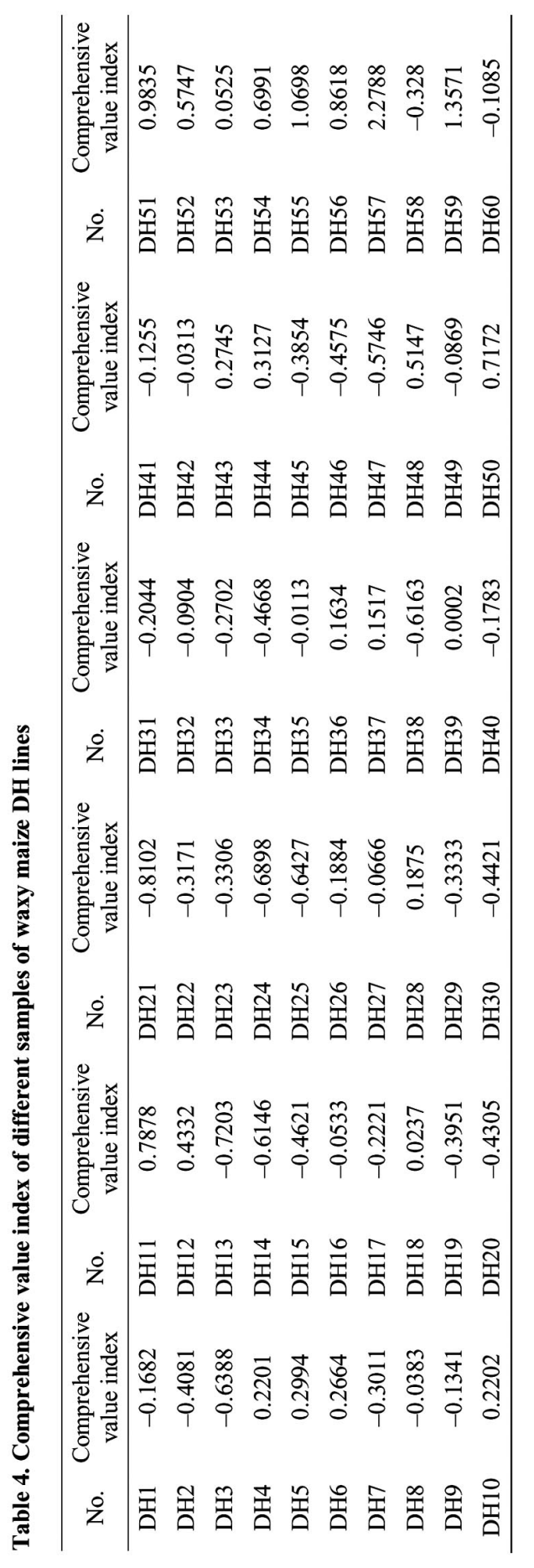




\section{Acknowledgements}

The authors gratefully appreciate the helpful comments and suggestions from Dr. Rengui Zhao and other anonymous reviewers. Thanks for PhD Research Foundation 2018[5002] and the Scientific Research Project Fund of Jilin Provincial Education Department (JJKH20190979KJ).

\section{References}

Beauregard M and Hefford MA 2006. Enhancement of essential amino acid contents in crops by genetic engineering and protein design. Plant Biotechnol. J. 4(5): 561-574.

Caňas RA, Quillere I, Chirst A and Hirel B 2009. Nitrogen metabolism in the developing ear of maize (Zea mays): analysis of two lines contrasting in their mode of nitrogen management. New Phytol. 184(2): 340-352.

Dong ZS, Lu H, Chai YC and Cai Z 2015. Concept and practices of maize commercial breeding in China. J. Maize Sci. 23: 1-9.

Galili G and Amir R 2013. Fortifying plants with the essential amino acids lysine and methionine to improve nutritional quality. Plant Biotech. J. 11(2):211-222.

Gong KJ, Liu ZX and Chen LR 2012. Discussion on the fresh waxy corn served as staple food. Chinese Agric Sci Bulletin, 28(36): 312-316.

Hao XQ, Wu ZK and Zhao G 2008. Studies on the amino acid content of sweet-waxy maize kernels for fresh food. J. Maize Sci. 16(6): 62-67.

Huang YB and Rong TZ 1998. The genetic diversity and evolutionary origins of waxy maize germplasm resources in China. Crops (supplement), pp. 77-80.

Jiang L, Ci JB, Cui XY and Zhang Y 2014. Study on induction rate and doubling rate of maize haploid under different ecological conditions. J Jilin Agric Univ 36(2): 139-143.

Ke YP, Shi HC and Niu YZ 2006. Genetic and combining ability analysis of nutritional quality traits in maize. J Sichuan Univ: Natural Sci. 43(5): 1146-1153.

Li RX, Yin JF and Shen SD 2011.Analysis of free amino acid composition in Porphyra yezoensis. Amino Acids \& Biotic Resources 33(1): 4-9.

Lindroth P and Mopper K 1979. High Performance Liquid Chromatographic Determination of Subpicomole Amounts of Amino Acids by Precolumn Fluorescence Derivatization with $o$-Phthaldialdehyde. Analytical Chemistry 51(11): 1667-1674.

Liu P, Lu WP and Lu DL 2009. Quality differences and physicochemical index screening for quality evaluation in waxy corn. J. Yangzhou Univ. (Agric and Life Sci. edn.) 30(3):16-21.

Liu RS, Yang HQ and Huang YW 2007. Extraction, purification and analysis methods of free amino acid in the plant. J of Henan Univ of Sci and Tech: Natural Sci. 28(3): 76-79.

Massart DL, Vandeginste BGM, Buydens LMC, de Jong S, Lewi PJ, Smeyers-Verbeke J and Mann CK 1998. Handbook of Chemometrics and Qualimetrics. Applied Spectroscopy 52(8): 302A.

Qi X, Zhao YJ and Liang J 2001. Analysis of heterosis on amino-acids of maize. J. Jilin. Agric. Univ. 23 (2): $17-20$.

Seebauer JR, Moose SP and Fabbri BJ 2004. Amino acid metabolism in maize earshoots. Implications for assimilate preconditioning and nitrogen signaling. Plant Physiol. 136(4): 4326-4334.

Shi ZS, Li FH and Wang ZB 2002. Study on current situation and problem of fresh eating research and development. J. Maize Sci. (supplement) 10: 93-96.

Sun LJ, Guo YR and Li JJ 2012. Analysis of regional characteristics of free amino acids in pulp of "Nagafu2" apples from different habitats. Food Sci. 33(5): 539-557.

Wang M, Zhang JS and Zhao YL 2010. Determination of free amino acids content in corn syrup by HPLC. China Brewing 5(218): 156-159.

Wang QD and Yang J 2010. Food experimental design and statistical analysis. pp. 343-366. Beijing, China. 
Wenefrida I, Utomo HS, Blanche SB and Linscombe SD 2009. Enhancing essential amino acids and health benefit components in grain crops for improved nutritional values. Recent Patents on DNA \& Gene Seq. 3(3): 219-225.

Yang BM, Yao LX and Guo OB 2011. Analysis of free amino acids in litchi fruits from different cultivars. Food Sci. 32(16): 249-252.

Zhang SH, Yi HH and Cai ZR 2008. Advances in research on waxy maize germplasm. J. Maize Sci. 16(3): 44-46.

Zhao T, Shi HZ and Ji XM 2011. Difference in composition and content of free amino acids among various tobacco types. Acta Tabacaria Sinica 17(2): 13-17.

(Manuscript received on 16 July, 2018; revised on 26 March, 2019) 